$P_{1}=-m$

\title{
Las Competencias de Comunicación Intercultural en la Empresa Global y su Desarrollo en el Aula de Español para Fines Económicos y Comerciales.
}

\author{
Luís V. Gómez García ${ }^{\mathrm{a}}$, Paulo Silveira ${ }^{\mathrm{a}}$, Margarida Morgado ${ }^{\mathrm{a}}$ \\ ${ }^{a}$ Instituto Politécnico de Castelo Branco (PORTUGAL); \\ idaluisproyectos@gmail.com
}

\begin{abstract}
Resumen
La presente investigación surge en el ámbito del proyecto financiado por la Unión Europea a través de la agencia Erasmus +, ICCAGE-Intercultural Communicative Competence: an advantage for global employability (http://iccageproject.wixsite.com/presentation), cuya finalidad es, mediante el incremento de la competencia comunicativa intercultural de los estudiantes de la educación superior de Portugal, España, Hungría y la República Checa, mejorar los índices de inserción laboral de los recién graduados de estos cuatro países especialmente damnificados por el desempleo juvenil.

Pretendemos con este estudio determinar qué competencias de comunicación intercultural empresarial son requeridas en la actualidad por los empleadores internacionales, y averiguar cuál es el modo más adecuado de activarlas e incorporarlas a los estudiantes de español para fines económicos y comerciales (ELEFEC) en la enseñanza superior.

El instrumento de recogida de datos destinado a averiguar cuál es el papel que juega en nuestros días la comunicación empresarial internacional, es un cuestionario/entrevista que fue creado por un conjunto de especialistas en comunicación intercultural empresarial de Hungría, España, Portugal y la República Checa. El cuestionario/entrevista se aplicó, entre noviembre de 2015 y enero de 2016 , a un total de 28 empleadores que desarrollaban su actividad profesional en empresas de ámbito internacional.

Los datos obtenidos nos permiten identificar cuáles son las competencias en comunicación empresarial internacional imprescindibles para integrarse con éxito en los contextos multiculturales y multilingües que caracterizan a las empresas actuales, así como averiguar cuál es la metodología más adecuada para desarrollar estas destrezas, conocimientos, actitudes y conciencia crítica intercultural en el marco de la enseñanza y aprendizaje de ELEFEC en la educación superior.
\end{abstract}

Keywords: Español como lengua extranjera para fines económicos y comerciales, Comunicación intercultural para fines profesionales, Competencia comunicativa intercultural. 


\section{Introducción y Objetivos de la Investigación}

Las sociedades del actual capitalismo tardío se caracterizan por su fuerte competitividad, sus mercados globales y cambiantes - lo que implica una enorme movilidad laboral- y por sus equipos multiprofesionales y multinacionales. Todo ello exige a sus trabajadores, además del dominio de lenguas extranjeras, capacidades de negociación de la diversidad cultural y de reflexión crítica sobre sus complejos sistemas de organización y comunicación.

En este contexto, la competencia comunicativa intercultural -entendida como el conjunto de habilidades para interactuar apropiadamente entre lenguas y culturas- emerge como un factor fundamental para enfrentar los desafíos producidos por la internacionalización de la producción, del empleo y de las empresas 1 .

Así pues, para lograr una efectiva integración en el competitivo mercado laboral global que define a nuestras sociedades, debemos, en primer lugar, identificar cuáles son las competencias de comunicación intercultural más demandadas en la actualidad por los empleadores internacionales y, posteriormente, descubrir cuál es la manera más adecuada de desarrollarlas en los futuros trabajadores del área del comercio y la empresa.

Son estos los dos objetivos que pretendemos alcanzar en esta investigación, encuadrada en el proyecto financiado por la unión europea a través de la agencia Erasmus + ICCAGE-Intercultural Communicative Competence: an advantage for global employability, (http://iccageproject.wixsite.com/presentation) cuya finalidad es, mediante el incremento de la competencia comunicativa intercultural de los estudiantes de educación superior de Portugal, España, Hungría y la República Checa, mejorar los índices de inserción laboral de los recién graduados de estos cuatro países, especialmente afectados por el desempleo juvenil.

A continuación, describimos el diseño metodológico adoptado en este estudio, presentamos los resultados obtenidos y los analizamos críticamente, de acuerdo con su potencial de aplicación a la enseñanza y aprendizaje de ELEFEC (Español como Lengua Extranjera para Fines Económicos y Comerciales) en el contexto de la educación superior. ${ }^{2}$

\section{Diseño Metodológico}

El instrumento de recolección de datos utilizado en el presente estudio fue un cuestionario/entrevista. Se envió para ser completado por correo electrónico y, posteriormente, se usó en entrevistas cara a cara. En consecuencia, esta investigación asume un carácter no-experimental, cualitativo y transversal. Por su parte, el muestreo es no probabilístico y por conveniencia.

El cuestionario/entrevista destinado a averiguar cuál es el papel que, según los empleadores internacionales, juega en nuestros días la comunicación empresarial, fue creado mediante la

\footnotetext{
${ }^{1}$ Los resultados de diversas investigaciones empíricas, entre las que destacan los proyectos INTENT (2007-2009) y C-EO Coach for Entrepreneurial Opportunities (2013-2015), corroboran que el desarrollo de la competencia comunicativa intercultural de los profesionales se constituye como un elemento esencial para una eficaz comunicación, externa e interna, en las empresas internacionales de hoy en día.

${ }^{2}$ La necesidad de contribuir a la mejora de la enseñanza y el aprendizaje de ELEFEC que se persigue en este artículo, no se justifica únicamente a partir de nuestra experiencia docente, sino que también se evidencia en estudios de referencia. Así, al revisar los actuales enfoques pedagógicos de ELEFEC, Lieve Vangehuchten y Ana Moreno Bruna (2015:76) concluyen que los materiales de aprendizaje elaborados en este ámbito formativo, "siguen dirigiéndose a su público meta como si fueran a funcionar en un contexto laboral exclusivamente español, lo cual no es en absoluto el caso para la mayoría de ellos, ya que el público ELEFEC tiene un marcado perfil internacional”
} 
metodología de grupos de discusión por 9 especialistas en comunicación intercultural empresarial de Hungría, España, Portugal y República Checa. Para ello se efectuaron, entre septiembre y octubre de 2015, 5 reuniones por Skype, con una duración media de 50 minutos. La versión final del cuestionario/entrevista, obtenida por consenso, se pilotó en un grupo de 8 empleadores para analizar la claridad y comprensión de las preguntas.

Entre noviembre de 2015 y enero del 2016 se aplicó el cuestionario/entrevista a 28 empleadores que desarrollan su actividad profesional en empresas de ámbito internacional: 10 en Portugal, 6 en cada uno de los restantes países.

Además de una primera pregunta acerca de los datos de cada empleador, enumeramos a continuación los tópicos que vertebran el cuestionario/entrevista destinado a determinar qué competencias de comunicación intercultural empresarial son requeridas en la actualidad por los empleadores internacionales, así como a averiguar cuál es el modo más adecuado de activarlas e incorporarlas a los estudiantes de ELEFEC en la enseñanza superior:

1. La diversidad cultural y la comunicación intercultural existentes en la empresa.

2. La preparación que en el campo de la comunicación empresarial intercultural ofrece el EEES (Espacio Europeo de Educación Superior)

3. Los consejos de los empleadores para trabajar con éxito en empresas internacionales.

4. Las competencias fundamentales para integrarse de forma efectiva en el mercado laboral global.

\section{Resultados}

A continuación presentamos los resultados más significativos conseguidos tras la aplicación de los cuestionarios/entrevistas.

Los 28 empleadores internacionales participantes indican que establecen comunicación empresarial de forma frecuente con socios y clientes de una gran variedad de culturas, como se aprecia en la siguiente tabla, que recoge una parte significativa de los países mencionados.

\begin{tabular}{lllcc}
\hline Europa & Africa & Asia & $\begin{array}{c}\text { Norte America \& } \\
\text { Oceanía }\end{array}$ & $\begin{array}{c}\text { América central } \\
\text { y del sur }\end{array}$ \\
\hline Francia & Egipto & Rusia & EEUU & Brasil \\
Austria & Sudáfrica & Mongolia & Canada & Argentina \\
Eslovenia & Namibia & Georgia & Australia & Costa Rica \\
Alemania & Etiopia & Filipinas & México \\
Grecia & Mozambique & Indonesia & Ecuador \\
Italia & Angola & Timor Este & Chile \\
Suiza & Marruecos & Vietnam & Perú \\
Polonia & Cabo Verde & China & Otros... \\
España & S. Tomé \& & Japón & \\
Irlanda & Príncipe & Tailandia & \\
Republica Checa & Otros... & India & \\
Eslovaquia & & Turquía & \\
Francia & & Jordania & \\
Noruega & Israel & \\
Ucrania & Siria & \\
Otros... & & Otros... & \\
\hline
\end{tabular}

Tabla 1. Países con los que los empleadores establecen comunicación empresarial.

Además, los empleadores entrevistados afirman que la comunicación con este diverso conjunto de socios y clientes internacionales se realiza tanto a través de los medios on-line, especialmente por email y video conferencia, como de forma presencial, para llevar a cabo reuniones de trabajo, proyectos 
desarrollados en equipos internacionales, presentaciones de productos, contratos, informaciones técnicas, etc.

Verificamos convergencia en las respuestas relativas al segundo tópico del cuestionario-entrevista, en el sentido de que, según casi todos los empleadores internacionales de los cuatro países consultados, la educación superior no prepara adecuadamente a los estudiantes para trabajar en contextos empresariales multilingües y multiculturales. Carecen los estudiantes de la enseñanza superior, al entender de los empleadores participantes en este estudio, de competencias esenciales para integrarse con éxito en la economía globalizada de hoy en día, en especial de sensibilidad ante las diferencias culturales y de habilidades de comunicación intercultural. No obstante, algunos empleadores advierten que los recién graduados que poseen experiencia de interacción efectiva con personas de otras culturas - por haber participado en programas de movilidad académica o por haber trabajado fuera- están mejor preparados para trabajar eficazmente en contextos internacionales que los que nunca vivieron en el extranjero.

Con respecto a las recomendaciones que los empleadores hacen a los trabajadores que, en el ámbito profesional y personal, tendrán que interactuar con personas de otras culturas, tres palabras aparecen reiteradamente en sus respuestas: flexibilidad, adaptación y experiencia. Términos que convergen en la idea de que, para lograr una comunicación empresarial exitosa, es necesario realizar una adaptación efectiva al contexto específico en el que se llevará a cabo el encuentro intercultural. Por esta razón, los empleadores aconsejan analizar todos los factores presentes en la interacción intercultural, siendo los más referidos el estilo de comunicación del interlocutor, el conocimiento sobre la historia y las relaciones sociales que determinan la cultura del otro participante en la interacción, y la terminología específica del área empresarial. Sea cual sea la interacción cultural del encuentro, insisten los empleadores en la necesidad de que los trabajadores muestren flexibilidad a la hora de gestionar las diferencias culturales con el socio o cliente.

Otro consejo que los empleadores mencionan con frecuencia es el de que los empleados acudan a los encuentros interculturales acompañados por personas con experiencia en negociaciones con la cultura específica con la que se va a llevar a cabo la interacción profesional y personal.

En el último de los temas abordados en las entrevistas-cuestionarios sobre la comunicación empresarial internacional, el relativo a las competencias que necesitan los jóvenes graduados para enfrentar con éxito las demandas del mercado laboral actual, multicultural y multilingüe, hemos clasificado las competencias referidas por los empleadores internacionales en cuatro grupos, siguiendo las directrices de Byram (1997) acerca de los componentes que conforman la competencia comunicativa intercultural. Así, en una categorización que facilita la compresión de cómo se articula en la actualidad la comunicación empresarial intercultural, referimos en la siguiente tabla las competencias que expresan destrezas, conocimientos, conciencia cultural crítica y actitudes.

DESTREZAS
Comunicar eficazmente en lenguas extranjeras
Estar preparado para la CI (comunicación intercultural)
Adaptarse a diferentes contextos
Adaptar el lenguaje a diferentes tipos de textos, situaciones y registros
Escuchar e interpretar con precisión los hábitos de otras culturas
Resolver adecuadamente conflictos
Utilizar herramientas TIC
Colaborar en proyectos (destrezas de trabajo en equipo)
CONOCIMIENTOS
Estudios culturales y religiosos
Cómo evitar los preconceptos y equívocos
Historia
La experiencia de vivir fuera de su país de al menos seis meses
Psicología social
CONCIENCIA CULTURAL CRÍTICA




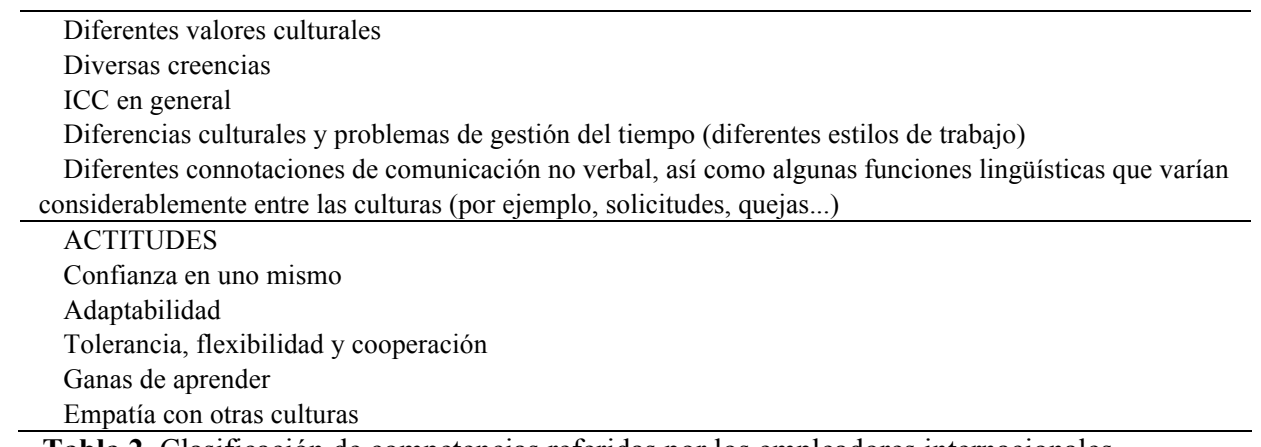

Tabla 2. Clasificación de competencias referidas por los empleadores internacionales

\section{Análisis de los Resultados}

La convergencia mostrada por los empleadores internacionales a la hora de referir que la enseñanza superior no proporciona a los recién graduados la preparación necesaria para encarar adecuadamente las demandas del actual mercado laboral globalizado, justifica la pertinencia de analizar los datos obtenidos en este estudio, con lo cual esperamos contribuir a mejorar la formación que en el campo de la comunicación empresarial intercultural ofrece en la educación superior el ELEFEC. Asimismo, la constatación de que la actual comunicación empresarial se caracteriza por la elevada diversidad cultural de las situaciones y contextos en que se produce, nos lleva a concluir que el aprendizaje y la enseñanza de ELEFEC debe ser necesariamente encarada desde una perspectiva intercultural.

Los datos obtenidos nos permiten alcanzar el primero de los dos objetivos de esta investigación: definir cuáles son las competencias de comunicación empresarial intercultural que los estudiantes de ELEFEC en la enseñanza superior necesitan adquirir para una eficaz adaptación al mercado global de nuestros días. A este respecto, la clasificación de competencias que hemos realizado en destrezas, conocimientos, actitudes y conciencia cultural crítica, además de ayudar a una mejor comprensión de los factores que determinan la actual comunicación empresarial internacional, puede contribuir a la mejora del diseño de cursos de ELEFEC, que suelen estar centrados en aspectos lingüísticos y en terminología del área de los negocios únicamente del ámbito hispanohablante.

Así, y frente a las programaciones de enseñanza y aprendizaje de lenguas para fines específicos del pasado, que incidían en la formación en contenidos, en los últimos años hemos asistido a un cambio significativo en el espacio europeo de educación superior: los objetivos de aprendizaje de un curso, asignatura, etc. han pasado a establecerse en términos de competencias. La formación en competencias no solo determina a nivel profesional quién es más apto para un puesto de trabajo, sino que además identifica qué recursos son necesarios para actuar eficazmente en situaciones específicas de un ámbito laboral (Vinagre Laranjeira 2010, p 33). Podemos responder a estos dos aspectos, preparando así a nuestros estudiantes para responder eficazmente a las demandas del actual mercado laboral, mediante la introducción en las programaciones ELEFEC de las competencias de comunicación empresarial intercultural -destrezas, conocimientos, actitudes y conciencia cultural crítica - identificadas por los empleadores consultados como necesarias para conseguir un buen desempeño en escenarios multinacionales.

Una vez determinadas cuáles son las competencias en comunicación empresarial internacional imprescindibles para integrarse con éxito en los contextos multiculturales y multilingües que caracterizan a las empresas actuales, así como justificada la necesidad de incluirlas en el diseño curricular de ELEFEC, nos centramos en averiguar, con base en los datos obtenidos, cuál es la metodología más adecuada para desarrollar estas destrezas, conocimientos, actitudes y conciencia crítica intercultural en el marco de la enseñanza y aprendizaje de ELEFEC en la educación superior. 
Tanto si los empleadores esperan que los trabajadores adquieran la formación necesaria para actuar de manera eficaz y competente en su puesto de trabajo mediante la experiencia en situaciones auténticas de comunicación empresarial, como si aconsejan a los empleados acudir a los encuentros de negocios acompañados por personas experimentadas en la interacción cultural específica que se llevará a cabo, lo más relevante de los resultados obtenidos es que la experiencia se constituye como el factor fundamental a la hora de adquirir y desarrollar las competencias necesarias para efectuar una exitosa comunicación empresarial intercultural.

De ello se desprende que la forma más adecuada de activar y desenvolver la competencia comunicativa intercultural empresarial en los estudiantes de ELEFEC, es la exposición de estos futuros profesionales a escenarios de interacción empresarial internacional. Recomendamos, pues, adoptar un enfoque didáctico experiencial en las clases de ELEFEC, lo cual supone la inmersión de los estudiantes en contextos multilingües y multinacionales, donde se requiere un uso efectivo de las competencias de comunicación empresarial intercultural identificadas por los empleadores participantes en este estudio.

Para materializar en el aula de ELEFEC este enfoque experiencial de enseñanza y aprendizaje de comunicación empresarial intercultural, disponemos de un valioso elemento: la tele colaboración, la actividad de involucrar a los estudiantes en actividades comunicativas en línea. El aprendizaje colaborativo asistido por ordenador nos permite recrear los escenarios de comunicación internacional auténtica referidos por los empleadores, al tiempo que adecuamos la comunicación profesional a los contextos más frecuentes en que, como demuestran los resultados obtenidos, tiene lugar: los medios online.

Con la realización en el aula de ELEFEC de proyectos empresariales o comerciales en equipos internacionales de estudiantes y profesores conectados por tele colaboración, no solo generamos aprendizaje experiencial en los alumnos, sino que facilitamos el análisis comparativo de creencias y valores entre culturas, transmitimos conocimientos sobre los modos de vida social, activamos las habilidades de interacción intercultural, ayudamos al reconocimiento de que los estereotipos son impedimentos que obstaculizan la comunicación intercultural y las relaciones sociales y comerciales, etc...Favorecemos, en su conjunto, con este enfoque didáctico experiencial, la activación y el desarrollo de las competencias identificadas por los empleadores como fundamentales en la actual comunicación empresarial, y, en consecuencia, preparamos más adecuadamente a nuestros alumnos de ELEFEC para enfrentar los desafíos de un mundo y un mercado laboral cada vez más global.

\section{Referencias}

Byram, M. (1997). Teaching and Assessing Intercultural Communicative Competence. Clevedon: Multilingual Matters.

Byram, M., Gribkova, B. \& Starkey, H. (2002). Developing the Intercultural Dimension in Language Teaching. Strasbourg: Council of Europe.

Byram, M. (2008). From Foreign Language education to Education for Intercultural Citizenship. Clevedon: Multilingual Matters.

C-EO (2013-2015). Coach for entrepreneurial opportunities. In Europe. Lifelong Learning

Programme: Europe. Recuperado de: https://ec.europa.eu/budget/euprojects/coach-entrepreneurialopportunies-c-eo_en

Council of Europe (2016). Competences for Democratic Culture. Living Together as Equals in culturally diverse democratic societies. Strasbourg: Council of Europe. Recuperado de: http://www.coe.int/t/dg4/education/Source/competences/competences-for-democratic-culture_en.pdf

Dooly, Melinda (2006). Integrating Intercultural Competence and Citizenship Education into

Teacher Training: a Pilot Project. Citizenship Teaching and Learning 2(1).18-30.

Glaser, E., Guilherme, M., García, M. C. M. \& Mughan, T. (2007). Intercultural competence for 
professional mobility. Graz: ECML. Recuperado de:

http://archive.ecml.at/mtp2/publications/B3_Icopromo_E_internet.pdf. 11/11/2015.

González Piñero, M., Díaz, C. G., \& Vez, J. M. (2010). Didáctica de las lenguas modernas. Competencia plurilingue e intercultural. Madrid: Editorial Síntesis.

Guilherme, M. (2002). Critical Citizens for an Intercultural World. Foreign Languages Education as Cultural Politics. Clevedon: Multilingual Matters.

Holliday, A., Hyde, M. \& Kullman, J. (2004). Intercultural Communication. An Advanced Resource Book. New York: Routledge.

Intent (2008). International Entrepreneurship A comparative analysis of interviews on "Intercultural Intelligence (II) in international business" in Europe. Lifelong Learning Programme: Europe. Recuperado de:

https://lirias.kuleuven.be/bitstream/123456789/521692/1/V_ACTAS_CIEFE_DEF.pdf

Instituto Cervantes (2006). Plan curricular del Instituto Cervantes. Niveles de referencia para el español. Madrid: Biblioteca Nueva. Recuperado de:

http://cvc.cervantes.es/ensenanza/biblioteca_ele/plan_curricular/default.htm

Lewis, R. (2002). The Cultural Imperative: Global Trends in the 21st Century. London: Nicholas Brealey Publishing.

Liddicoat, A. J. \& Scarino, A. (2013). Intercultural Language Teaching and Learning. Chichester: Wiley-Blackwell.

Nikleva, D. G. (2012). La competencia intercultural y el tratamiento de contenidos culturales en manuales de español como lengua extranjera. Resla 25, 165-187.

O'Dowd, R., \& Lewis, T. (eds) (2016). Online Intercultural Exchange: Policy, Pedagogy, Practice. Routledge: NY.

Riutort Cánovas, A. (2010). La competencia comunicativa intercultural en el aula de español de los negocios. Análisis de materiales para su enseñanza. Tesis doctoral inédita. Recuperado de: http://oa.upm.es/10621/1/ANTONIO_RIUTORT_CANOVAS_1.pdf

Trompenaars, F. \& Hampden-Turner, C. (1998). Riding the Waves of Culture: Understanding Diversity in Global Business. New York: McGraw-Hill.

Trompenaars, F. \& Wooliams, P. (2003). Business across Cultures. West Sussex: Capstone Publishing Ltd.

Tlang Proyect (2014) Superdiversity. Recuperado de: http://www.birmingham.ac.uk/generic/tlang/methodology/index.aspx

Unesco (2013). Intercultural Competences. Conceptual and Operational Framework. Paris: UNESCO. Recuperado de: http://unesdoc.unesco.org/images/0021/002197/219768e.pdf

Vangehuchten, L. \& Moreno Bruna, A. (2015). La enseñanza del español para fines económicos y comerciales hoy en día: hacia un nuevo diseño del curso ELEFEC. En El español lengua de la comunicación profesional. Artículos seleccionados del v congreso internacional de español para fines específicos (CIEFE V). Barcelona: Ministerio de Educación y Ciencia. Recuperado de: http://cvc.cervantes.es/ensenanza/biblioteca_ele/ciefe/pdf/05/cvc_ciefe_05_0001.pdf

Vinagre Laranjeira, M. (2010). Teoría y práctica del aprendizaje colaborativo asistido por ordenador. Madrid: Editorial Síntesis.

Vilá Baños, R. (2005). La competencia comunicativa intercultural. Un estudio en primer ciclo de la ESO. Tesis Doctoral. Barcelona: Universidad de Barcelona. Recuperado de:

https://dialnet.unirioja.es/servlet/tesis?codigo $=3646$

Esta publicación ha sido posible gracias al apoyo del programa Erasmus + en el ámbito del proyecto ICCAGE, cuyo número de referencia es 2015-1-CZ01-KA203-013992.

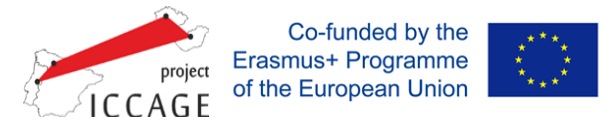

